\title{
Una aproximación al estudio de la conversación de la persona con alzheimer y sus interlocutores sanos
}

\author{
Beatriz Valles \\ Universidad Pedagógica Experimental Libertador, Venezuela
}

\begin{abstract}
Resumen
Esta investigación se propuso estudiar bajo un enfoque cualitativo y a partir de los aportes teóricos y metodológicos de la lingüística clínica, la conversación entre personas con demencia tipo Alzheimer y sus interlocutores sanos en el ámbito del hogar. Sus objetivos fueron: 1) Identificar los diferentes recursos conversacionales utilizados por todos los participantes; 2) Estudiar cómo el uso de estos recursos puede influir en la estructura de la conversación y 3) Analizar el uso de la gestualidad como vehículo de comunicación. Los informantes fueron dos personas con demencia tipo Alzheimer, sus respectivas cuidadoras y la investigadora. Se filmaron en vídeo, conversaciones en las cuales participaron todos, posteriormente se transcribieron y se analizaron. Los resultados muestran que las personas con demencia utilizaron la respuesta típica de pares adyacentes, repeticiones y paráfrasis. Se observó diferencia en la longitud de sus turnos y en el uso de oraciones complejas. Uno de los pacientes utilizó gestos en sustitución de palabras. Ambas cuidadoras emplearon repeticiones, adiciones de información y ajustaron su actividad lingüística. La investigadora utilizó marcadores y preguntas para iniciar la conversación, en forma de entrevista terapéutica. Los turnos de conversación se dieron de forma coordinada. Estos datos preliminares se consideran importantes para profundizar los conocimientos acerca de la conversación de la persona con Alzheimer y el rol que juegan sus interlocutores sanos en este proceso complejo y cambiante, que demanda actualmente un nuevo enfoque en su abordaje logopédico.

Palabras clave: Alzheimer; Análisis de la conversación; Demencia; Lingüística clínica.
\end{abstract}

\section{An approach to the study of the conversation between patients with alzheimer and their healthy interlocutors}

\begin{abstract}
This research aimed to study the conversation of people with dementia and their healthy interlocutors in the home context. The following objectives were proposed: 1) To analyze the variety of conversational resources (turns to speak, adjacent pairs, use of repairs and/or marks) used by all interlocutors (patients, caregivers and researcher); 2) To study how the use of these resources may or may not have an influence in the structure of the conversation; and 3) To value the use of other forms of non-linguistic communication, such as the use of gestures, for instance. The informants were two dementia patients, their caregivers and the researcher. In order to make the corpus, four linguistic interactions with all the participants were filmed in video during the period of one year. Some segments of the conversations were selected, transcribed into gloss and later analyzed. The results show that the dementia patients used discursive marks, the typical response of adjacent pairs, repetitions and paraphrases. When comparing both performances, we observed a marked difference in the length of their turns to speak and in the use of complex sentence structures. One of the patients used gestures as substitute for words. Both caregivers used repetition and addition of information, and adjusted their linguistic performance to the situation. The researcher used linguistic markers and questions to introduce the conversation, which had an influence on the organization of the speech, in a question-answer format, as in therapeutic or supervised interviews.
\end{abstract}


The turns to speak developed in an coordinated way. The contributions of this study are considered important to deepen our current knowledge on the conversation of patients with dementia and the role played by their regular interlocutor in this process.

Key words: Alzheimer; Dementia; Clinical linguistics; Conversation analysis.

Correspondencia con los autores: brvallesg@gmail.com

Recibido 01 Marzo 2013. Primera revisión 25 de junio de 2013. Aceptado 16 de julio de 2013.

Reconocimientos: Esta investigación contó con el apoyo del Fogarty Internacional Collaborative Research Training Grant (The University of Chicago-La Universidad del Zulia), del Laboratorio de Neurociencias (LUZ) y del Centro de Investigación del Departamento de Educación Especial del Instituto Pedagógico de Caracas (CIDEE-UPEL-IPC). Investigación aprobada por el Comité de Bioética de LUZ.

\section{Introducción}

La Demencia es un síndrome complejo de alteraciones intelectuales degenerativas que compromete el buen funcionamiento en múltiples áreas del desarrollo humano: psicosocial, cognitiva, lingüística, emocional, perceptiva y motora (Gaona, 2012). Los síntomas afectan al paciente y a todo su entorno personal: familia, compañeros, vecinos (Aldana 2009). La prevalencia de la demencia se incrementa con la edad, siendo esta de un $10,8 \%$ en personas entre 80 y 89 años y es más frecuente en mujeres, debido en gran medida a que ellas viven más tiempo (Maestre, 2007).

El lenguaje de las personas afectadas por la demencia manifiesta diversas características que han sido estudiadas por diferentes autores (Hamilton, 1994; Sabat, 2001; Ripich y Terrell, 1988; Orange, Lubinski y Higginbotham, 1996; Valles, 2009; Young, Manthorp y Howells, 2010), las cuales varían drásticamente de un paciente a otro y de acuerdo con la progresión y etiología de cada tipo de demencia. Las más resaltantes son la anomia, la parafasia, el uso de neologismos y de muletillas o palabras carentes de sentido, alteraciones en la longitud de las emisiones verbales y severas dificultades para seguir el tema de una conversación. Todos estos trastornos provocan una comunicación difícil entre los pacientes y sus interlocutores habituales.

La Enfermedad de Alzheimer es la principal causa de demencia (Young, et al., 2010). Maestre (2007) afirma que si bien en el cerebro de la persona con Alzheimer pueden encontrarse alteraciones estructurales conocidos como ovillos y placas, es la muerte de cierto tipo de neuronas (las colinérgicas y glutamatérgicas) lo que parece estar más relacionado con la demencia. Las neuronas colinérgicas producen acetilcolina, este neurotransmisor es imprescindible para la memoria y el aprendizaje. "Al verse afectada la producción de acetilcolina, los procesos de memoria y aprendizaje se ven fuertemente deteriorados, y el proceso de demencia se acentúa” (p.28). Por esta razón, la competencia comunicativa de la persona con Alzheimer (en adelante PA) comienza a 
trastornarse de manera progresiva a medida que los cambios en la estructura cerebral provocan deterioro cognitivo y por consiguiente, numerosos problemas lingüísticos, relacionados con las fallas en el uso del lenguaje tanto en forma oral como escrita, las cuales ser resumidas en limitaciones con recordar o seleccionar la palabra correcta, deletrear o utilizar la gramática apropiada (McKhann et al., 2011). Desde la fase inicial se manifiestan dificultades para evocar información y en consecuencia seguir de manera eficiente la conversación (Rumbos y Valles, 2010), las fallas en la memoria semántica provocan que la misma se vea limitada, hasta llegar a un grave compromiso lingüístico (Bayles, Tomoeda, Cruz y Mahendra, 2000).

La PA muestra dificultades para comprender, por lo que no llega a ajustarse a los rápidos cambios contextuales y en consecuencia puede reducir sus turnos de habla, o hablar fuera de contexto. Las limitaciones descritas aumentan las dificultades en la competencia discursiva, especialmente cuando su interlocutor no pertenece al contexto diario y no conoce la historia de vida del paciente, lo que no le permite inferir sobre la temática o anticipar las palabras o conceptos que la PA no es capaz de evocar.

De acuerdo con lo anterior, podemos concluir que la PA presenta un trastorno en la expresión y comprensión del lenguaje; en consecuencia su rendimiento lingüístico lo aparta de la norma que utilizan los adultos mayores que tienen un proceso de envejecimiento normal. Las limitaciones lingüísticas más resaltantes se observan en el nivel léxico-semántico, lo que hace su comunicación difícil, especialmente a partir de la etapa media y avanzada de este síndrome. El trastorno se evidencia en un hecho objetivo: la reducción de la coherencia y las limitaciones en el uso de los recursos cohesivos. Situación que debe ser compensada por su interlocutor a través de diferentes estrategias, para poder construir de manera adecuada y cooperativa la coherencia del discurso durante la conversación.

Gallardo-Paúls (2005) afirma que el interlocutor clave, es decir la persona que habitualmente atiende a un paciente (en el caso de sus investigaciones con personas con Afasia), tiene mayores oportunidades de comunicarse con él. Para la PA esta situación de dependencia con su interlocutor clave se profundiza pero presenta matices que aún no han sido suficientemente analizadas, especialmente con lo relacionado a cómo se gestiona la comunicación con este tipo de paciente en diversos contextos. A este respecto es importante señalar que el impacto que el diagnóstico y el deterioro cognitivo provoca en los familiares de una PA puede generar en sus interlocutores sanos una gestión de la conversación bastante limitada. Tal como apunta Hernández (2012) “el 
déficit lingüístico puede alterar profundamente las imágenes o identidades sociales, tanto de los sujetos portadores del déficit como de sus interlocutores" (p. 50).

Siguiendo este orden de ideas, Young, Manthorp y Howells (2010) advierten que tradicionalmente en los espacios clínicos algunos profesionales manifiestan actitudes negativas con respecto a interactuar con estos pacientes. Por ejemplo, muchos enfermeros consideraban algo verdaderamente excéntrico el desear hablar con una PA. Cabría investigar si estas creencias se dan en otros integrantes de los equipos de salud encargados de la atención de esta población, como por ejemplo en los logopedas, motivado a que actualmente los servicios dirigidos a esta población son escasos y la formación de estos profesionales no profundiza en el diseño de programas logopédicos dirigidos a la misma.

Hoy en día la situación a la que se enfrentan los profesionales responsables de evaluar e intervenir la comunicación de la PA está determinando nuevos rumbos de acción no sólo en la forma como se planifica su atención, sino en los métodos que se utilizan para investigar en esta población (Hamilton, 1994; MacKay, 2007; Valles, 2008). La necesidad creciente de atender personas afectadas por algún trastorno del lenguaje y de analizar sus intercambios comunicativos con sus interlocutores habituales, ha dado impulso a la lingüística clínica, disciplina que se encarga del análisis y evaluación de la conducta verbal en patologías del lenguaje (Hernández y GallardoPaúls, 2010). Sus aportes han permitido estudiar la comunicación de la PA en otra perspectiva, pues desde la pragmática clínica los investigadores abordan el estudio del habla patológica utilizando los métodos de la pragmática, lo que ha tenido un impacto positivo en la práctica en los contextos clínicos y en el estudio de la interacción verbal humana en general (Gallardo-Paúls, 2005).

Esto se ha hecho evidente en el campo de la Afasia, área en donde los investigadores se han interesado por analizar la conversación y valorar la actividad de los diferentes actores, como por ejemplo, pacientes e interlocutores claves (GallardoPaúls, 2007; Moreno, 2010; Pietrosemoli, 2007; Tinti y Marogna, 2000). A este respecto cabe destacar que los hallazgos de Gallardo-Paúls (2007) muestran cambios en el ritmo conversacional, uso de comunicación no verbal, estrategias de facilitación de la participación del paciente (preguntas polares, de inventario cerrado) y para facilitar la comprensión del paciente. Por otra parte, los aportes de Pietrosemoli, Vera, González y Coutín (2005) señalan el uso de marcadores discursivos por parte de los pacientes y de reparaciones conversacionales entre éstos y sus logopedas (Pietrosemoli, 2007). Ambas 
investigadoras concluyen que el $\mathrm{AC}$ es una herramienta útil para conocer el impacto de la afasia sobre la capacidad de conversar y para diseñar los programas de intervención logopédica.

Investigaciones desarrolladas en los últimos quince años han analizado la comunicación de la PA y otras personas (cuidadores, proveedores de salud) no como una actividad individual, sino que se ha enfocado la atención en la actividad lingüística de todos los participantes de los intercambios. (Brauner y Merel, 2006; Malagón, Rodríguez, Hernández y Pardo, 2005; Jaramillo, 2010; Valles, 2011). Los datos aportados en estos estudios han enfatizado la necesidad de estudiar la comunicación en una perspectiva más amplia, pues la ejecución comunicativa del paciente se ve influida por la de su interlocutor sano, quien puede ayudarlo a mantenerse dentro de la conversación o reducir su interacción con él hasta eliminarla (Tomoeda 2001; Orange, Lubinski y Higginbotham, 1996).

Este cambio ha motivado que la actuación del logopeda se vea enfrentada a nuevas exigencias. Quizás la principal sea el tener que planificar una intervención dirigida a ralentizar los efectos del deterioro cognitivo sobre la actuación lingüística del paciente, centrando sus esfuerzos en el cuidador y los demás especialistas o personas que atienden a la PA. Sin embargo aún es necesario investigar más sobre cómo se gestiona el error en el acceso al léxico o la ampliación del tiempo en los turnos de habla, entre otros aspectos que pueden alterar la interrelación comunicativa entre PA y sus interlocutores sanos.

Hasta el presente la conversación de las PA se ha analizado mayoritariamente en residencias para adultos mayores (Hamilton, 1994) o en clínicas (Brauner y Merel, 2006). Sin embargo el estudio de la conversación en contextos más naturales, como por ejemplo en el hogar, ha sido escaso. Consideramos que este es el mejor escenario para analizar los intercambios lingüísticos, pues es allí donde la vida de la persona con demencia transcurre y la actividad del cuidador puede significar un elemento que soporte y ayude al paciente. En el hogar podrían obtenerse datos más significativos y reducirse lo que en opinión de Díaz (2010) es una limitación habitual en los contextos logopédicos, refiriéndose a la "supuesta pérdida de naturalidad en unas prácticas conversacionales que de manera sistemática y entusiasta se defienden como naturales" (pág. 45).

La información sobre el proceso de comunicación y el rol de todos los actores es necesaria para poder conocer cómo la demencia impacta al paciente, quien ve reducidas 
sus habilidades para comprender y expresarse correctamente, y a su interlocutor clave, quien como ya se dijo, puede realizar menores intentos para comunicarse con él y además se adaptan a este nivel de rendimiento llegando a reducir la longitud de los enunciados o a utilizar sólo preguntas cerradas o palabras aisladas en su conversación (Hamilton, 1994). Todos estos datos sirven de base para la elaboración de un plan de atención logopédica que debe orientarse a optimizar los intercambios lingüísticos a través de diferentes recursos. En función de ofrecer un tratamiento de calidad, el logopeda debe que orientar su acción sobre los diferentes actores, por esta razón se aconseja incluir en el proceso de diagnóstico el estudio de la conversación como vía para recabar datos que permitan desarrollar acciones más pertinentes en los espacios clínicos y además tener en cuenta que la atención de la PA, lo enfrenta a exigencias diversas que precisan la búsqueda de nuevos recursos para definir una práctica compleja que pueda ofrecer soluciones adaptadas a las necesidades comunicativas de la PA y de sus interlocutores habituales.

A partir de esta perspectiva, la presente investigación se propuso estudiar la actividad lingüística de la PA, incluyendo la participación del cuidador primario (interlocutor clave) y de la investigadora quien propició la conversación en los encuentros. Para alcanzar este objetivo se diseñó una investigación cualitativa y se centró la atención en analizar los intercambios que se dan en situaciones fuera del espacio clínico (consultorio, gabinete). Para esto se realizaron visitas a los hogares de dos PA y se filmaron diferentes conversaciones en las cuales participaron todas las personas ya señaladas. Posteriormente estas interacciones fueron transcritas y analizadas teniendo como referencia las orientaciones de Pietrosemoli (2007) para el análisis de conversaciones en poblaciones especiales. Cabe destacar que la intensión del análisis no estuvo dirigida al estudio estricto de la coherencia y cohesión de los textos conversacionales, sino en obtener información sobre las características de interacción comunicativa de los actores, datos que permitirán a futuro, orientar mejor las acciones de atención logopédica.

De acuerdo con esto, se estudió el uso de diversas estrategias comunicativas que emergieron en estos intercambios lingüísticos, y el papel del contexto en esta interacción. La investigación se planteó alcanzar los siguientes objetivos:

1) Identificar los diferentes recursos conversacionales utilizados por todos los participantes (pacientes, cuidadores e investigadora); 
2) Estudiar cómo el uso de estos recursos puede influir en la estructura de la conversación (turnos, pares adyacentes, uso de reparaciones y/o de marcadores) y

3) Analizar el uso de otras formas de comunicación, como por ejemplo la gestualidad.

Atendiendo los propósitos ya mencionados, a continuación se desarrollan una serie de conceptos relacionados con los mismos.

La conversación en las personas con demencia tipo Alzheimer: características y variaciones

Pietrosemoli (2007) define como conversación "a todo intercambio lingüístico que se establece entre dos o más hablantes de una misma lengua con propósitos comunicativos y de una manera que se desprende naturalmente de las circunstancias que rodean ese intercambio" (p. 307). Por su parte, Gallardo-Paúls (2005) afirma: "la manera más habitual de establecer contacto comunicativo con las personas que nos rodean es mediante la conversación; es el modo en que participamos en la vida social, y el modo en que creamos, mantenemos y reforzamos nuestras relaciones interpersonales" (p. 43). Al conversar utilizamos como instrumentos fundamentales para actuar los códigos lingüísticos, junto con otros códigos no verbales que acompañan a la palabra (Tusón, 2002).

La comunicación humana se establece con la conversación, a través de ella, emitimos opiniones, expresamos sentimientos, realizamos acuerdos (o desacuerdos) y planificamos actividades. Todos los participantes del intercambio contribuyen activamente (Brauner y Merel, 2006), de esta manera, lo que se dice es el resultado de una construcción que realizan todos, los aportes de todos los interlocutores son importantes, se pasa de un tema a otro con relativa facilidad y el contacto visual, volumen, entonación de la voz y el "lenguaje corporal" son elementos primordiales en la construcción del sentido (Gallardo-Paúls, 2005)

Para Grice (1975) toda interacción lingüística está regida por el principio de cooperación que se resume en cuatro máximas: 1) De cantidad: haz que tu contribución sea tan informativa como sea necesario (ni más ni menos) para las finalidades del intercambio; 2) De cualidad: Haz que tu contribución sea verdad. No digas lo que sabes que es falso; 3) De relación: Sé pertinente, no digas lo que no viene al caso y 4) De manera: Sé claro evita la ambigüedad, se breve sé ordenado. A partir de este principio los interlocutores evidencian su competencia comunicativa y su competencia lingüística, 
esta relación necesaria entre conocimiento pragmático y gramatical, es lo que permite ajustarse a las demandas del contexto y por supuesto a las necesidades y características de los interlocutores.

El tipo de conversación más frecuente es aquella que se desarrolla cara a cara, ocurre en un tiempo y lugar determinado, y los participantes actúan de manera coordinada con el propósito fundamental de compartir información. En ella la organización del sistema de turnos y otros aspectos están al servicio de la construcción del sentido conversacional (Tusón, 2002). De acuerdo con Gallardo-Paúls (1998), en las conversaciones cara a cara confiamos muchas funciones a la gestualidad, de esta manera, el uso que los hablantes hacen de una serie de gestos puede ser determinante para organizar su participación.

Desde una perspectiva psicológica es necesario considerar los procesos cognitivos que regulan el acto de conversar cara a cara, pues la atención, concentración, memoria y la capacidad de poder interpretar los gestos, posturas, inflexión de la voz y demás eventos que rodean este acto, acarrean una actividad cognitiva que obliga a coordinar diversas acciones y ajustarlas a los patrones que desde lo sociocultural se debe tener en cuenta, para garantizar que se cumplan las normas que pautan una conversación eficiente o exitosa.

La persona que sufre la enfermedad de Alzheimer o cualquier otro tipo de demencia, tiene problemas para participar con eficiencia en la conversación, las limitaciones en su atención y concentración provocan dificultades para leer el contexto y ajustarse a los cambios, turnos, variaciones del tema y otros eventos lingüísticos propios de estos intercambios. Por otra parte, en etapas avanzadas otros síntomas como la depresión, agresión y alucinaciones, deterioran aún más la calidad de la interacción pues provocan un fuerte impacto en el interlocutor sano, especialmente en sus cuidadores primarios (Aldana, 2009; Brewer, 2005).

De esta manera, el principio de cooperación se ve severamente amenazado en el caso de la demencia, pues esta afección se presenta mayoritariamente en adultos mayores, quienes están expuestos a una concepción sobre la vejez cargada de prejuicios de la sociedad, a la cual "se le suma la de profesionales que trabajan con viejos, aportando a la reproducción de este imaginario social una carga sumamente negativa “(Ludí, 2005. p. 27), que genera que el funcionamiento lingüístico del adulto mayor se perciba como limitado o equívoco. 
A lo ya expuesto se le agrega además el efecto negativo del diagnóstico, lo que genera una situación comunicativa altamente compleja, marcada por profundas limitaciones para el paciente. Varios autores (Petersen, 2004; MacKay, 2007; Jaramillo, 2010) alertan de que los cambios en la comunicación son los primeros indicios del trastorno cognitivo y de que además, estos signos no son tomados en cuenta por los familiares o por los proveedores de salud, hecho que determina que muchas veces el adulto mayor no sea diagnosticado tempranamente y en consecuencia, no reciba una atención oportuna.

El diagnóstico de la enfermedad de Alzheimer representa un fuerte golpe no sólo para el paciente sino para toda la familia, especialmente para el cuidador primario, quien se ve sumido en una fuerte exigencia: cuidar las 24 horas del día, los 365 días del año. Todas estas circunstancias han definido una nueva práctica asistencial en el ámbito logopédico, pues dado que el deterioro de la PA es progresivo el objetivo primordial de la intervención es ralentizarlo, es decir tratar de enlentecer el progreso del deterioro. Para alcanzar este objetivo la atención se debe focalizar no sólo en el paciente sino en el llamado interlocutor clave, es decir, en la persona que habitualmente lo atiende y más se relaciona con él.

Desde esta perspectiva, el análisis de la actividad de ambos interlocutores muestra que el intercambio lingüístico debe ser analizado más allá de la forma, por lo que los profesionales encargados del diagnóstico y la atención de las personas con trastornos del lenguaje deben prestar especial atención a la producción o acción lingüística de todos los actores involucrados. Savundranayagam, Hummert y Montgomery (2005) consideran necesario que al analizar la conversación de una persona que padece cualquier forma de demencia se profundice en el estudio de la actividad de ambos actores y se valore cómo el trastorno lingüístico y las percepciones que del paciente tiene el interlocutor sano, pueden afectar las posibilidades de un intercambio conversacional efectivo.

En esta línea es importante destacar que los intercambios entre logopedas y pacientes también deben ser analizados cuidadosamente, pues el contexto clínico conlleva a la gestión de la comunicación con algunas particularidades que lejos de apoyar una conversación fluida y efectiva, pueden limitarla. A este respecto cabe destacar los datos aportados por Díaz (2010), quien señala que la interacción clínica mantiene un carácter institucional que guía la selección de los temas y mantiene "una omnipresente asimetría entre profesional y paciente" (p. 46). 
Las PA pueden ser incluidas dentro del grupo población especial, definida por Pietrosemoli (2007) como aquellos individuos que por algún evento interno o externo, con evolución natural o presencia repentina, han padecido o padecen una alteración en algún aspecto de la capacidad lingüística normal, en forma permanente o transitoria. Esta misma autora asume como facultad lingüística normal a "la capacidad de cualquier persona para producir habla bien estructurada desde el punto de vista de la señal lingüística y que esté apropiadamente relacionada con el contexto en el que se produce" (p.305).

En otras palabras, cuando definimos como normal determinada producción lingüística, lo hacemos tomando en cuenta su contenido y forma, así como las posibilidades de actuación dentro de un determinado contexto. La norma provee las estrategias cohesivas consideradas adecuadas para lograr una coherencia tal que permita que una producción lingüística alcance su fin último: producir un efecto en el otro. El concepto de norma lingüística remite pues a las creencias que un grupo tenga con respecto a cómo deben producirse sus actos de habla, a su esquema cognoscitivo o ideológico con respecto al uso del lenguaje y con respecto a cómo concretar el uso de su lengua en determinados contextos. Por lo tanto, la función cognoscitiva es determinante para alcanzar una adecuada elaboración textual, esto explica el deterioro de la función lingüística de la PA.

Las limitaciones comunicativas de la PA corresponden en su mayoría al componente léxico-semántico, sin embargo es necesario destacar que ellas afectan el rendimiento lingüístico global de estos pacientes, por lo que aspectos referentes a la pragmática se verán involucrados. $\mathrm{Y}$ es que las variaciones referidas al proceso de acceso léxico, como por ejemplo las parafasias semánticas (sustitución de la palabra blanco por otra o por un neologismo), las perseveraciones (repetición de contenidos, muchos de ellos estereotipias verbales) o la anomia (problemas de acceso léxico o pérdida de palabras), acarrean dificultades en la organización sintáctica y en la continuidad del discurso (MacKay, 2007; Jaramillo, 2010).

El análisis de la conversación aplicado al estudio del intercambio lingüístico de la persona con demencia

El análisis de la conversación (AC) ha mostrado ser de gran utilidad para estudiar las características del intercambio comunicativo entre personas con demencias y sus cuidadores (Savundranayagam, Hummert y Montgomery, 2005). Este recurso permite 
valorar tanto la actividad lingüística del paciente como la del cuidador $\mathrm{u}$ otros interlocutores sanos en situaciones cotidianas de interacción. El interés del AC se orienta a describir las prácticas de interacción verbal y a explicar los roles que los hablantes asumen y que les permiten en última instancia regular la vida social.

Los actores en un acto de habla utilizan una serie de recursos con el objetivo de alcanzar un adecuado intercambio comunicativo. En el caso de la conversación cara a cara entre la PA y sus cuidadores ha sido posible determinar que estos últimos ajustan su propia ejecución teniendo en cuenta las dificultades del paciente. Por esto, reducen la longitud de los enunciados, seleccionan tópicos referidos a eventos concretos de la vida cotidiana (como por ejemplo las actividades de rutina: alimentación, aseo, paseos), parafrasean lo que dice el paciente o utilizan reparaciones indirectas (Valles, 2006).

El análisis de las características de la conversación de la PCD puede hacerse a partir de diferentes propuestas. Pietrosemoli (2007) sugiere analizar el discurso a partir del estudio de la conversación, teniendo como parámetros los elementos propios de este proceso, a saber: génesis, mecanismo y estructura. Para realizar esta actividad la autora insiste en valorar los siguientes aspectos: turnos en la conversación, los pares adyacentes, las reparaciones y los marcadores discursivos. A continuación se explicarán brevemente cada uno de estos elementos.

Los turnos en la conversación se refieren a las intervenciones individuales de cada participante en la conversación. Como se sabe, este proceso sigue una organización temporal y aunque en ocasiones los hablantes participan simultáneamente, casi siempre se hace posible alinear de forma secuencial sus intervenciones.

Se llaman pares adyacentes a las estructuras de obligación secuencial, es decir, que aparecen de forma coordinada en la cual la segunda en consecuencia de la primera. Por ejemplo: ¿Cómo estás?/ ¡Muy bien!; Buenas tardes/ Buenas.

Por su parte, la reparación conversacional es un complejo proceso que puede manifestarse de diversas maneras. Podemos identificar reparaciones dirigidas a corroborar el significado de un mensaje; este tipo de reparación es denominada indirecta y se relaciona directamente con la coherencia global de un texto.

Existen otros tipos de reparaciones dirigidas a solucionar los problemas relacionados con la forma del discurso; estas reparaciones son llamadas autoreparaciones y hetero-reparaciones. Las primeras son utilizadas por el hablante como una forma de auto-corrección. Las segundas son utilizadas por el interlocutor, quien corrige o completa determinado mensaje; en consecuencia, podemos establecer que este 
tipo de reparaciones está más relacionado con la cohesión porque las mismas tienen como objetivo reparar la forma del discurso utilizando en este proceso los elementos cohesivos, aunque en última instancia, ellas se relacionan e influyen también en la coherencia textual.

Los marcadores discursivos son aquellos elementos que establecen una secuencia en la comunicación (Schiffrin et al., 2001, citados por Pietrosemoli, 2007). Su función principal se dirige a organizar y estructurar el discurso y la interacción (Lesser y Milroy, 1993). Marcadores tales como y, pero y o, sirven para conectar ideas, información que precisa ser organizada siguiendo las reglas que la norma lingüística provee, es decir que en última instancia los marcadores le dan al discurso coherencia y cohesión.

El conocimiento actual sobre el uso de los aspectos ya mencionados por parte de los hablantes con demencia es insuficiente para conocer las características de su conversación, específicamente en el marco de la interacción con la PA. Por esto se propone una nueva orientación en el análisis que tome en cuenta las intenciones de todos los hablantes, el ambiente y contexto de la interacción y que explore profundamente cómo su uso está relacionado bien con la coherencia o bien con la cohesión del discurso, y cómo éstos ayudan a mantener o a construir una adecuada comunicación. En otras palabras, más que conocer el tipo de elemento: turnos, reparaciones, pares adyacentes o marcadores, interesa analizar, describir y explicar desde lo cualitativo, el impacto de su uso en la conversación en espacios sociales como el hogar.

También es importante analizar este proceso en función de poder determinar qué tipo de atención logopédica se requiere para mejorar la comunicación entre cuidadores primarios, proveedores de salud y personas con demencia, y conocer, si esto tiene un efecto positivo en el cuidado e intervención del paciente (Valles, 2010; Jaramillo, 2010). Sin embargo, es necesario destacar que este tipo de investigación presenta varias limitaciones, por una parte la práctica en el campo de la salud pública está arraigada en sus respectivos contextos culturales, por lo que hay que ser cuidadoso al generalizar los resultados, y por otra parte, el objetivo de este tipo de investigación está dirigido "a descubrir múltiples perspectivas y comprender sus repercusiones en la adopción de decisiones vinculadas con la salud" (Ulin, Robinson y Tolley, 2006, p. 5) .

A continuación se presenta el método utilizado en el presente estudio. 


\section{Objetivos de la investigación}

Partiendo de lo anteriormente expuesto, esta investigación se propuso estudiar la conversación de la PA, haciendo especial énfasis en el análisis de la actividad lingüística de todos los participantes, en este caso, dos pacientes con Alzheimer, sus cuidadores primarios y la investigadora, en el contexto de sus hogares y desde una perspectiva cualitativa.

Los objetivos propuestos fueron los siguientes: 1) Analizar los diferentes recursos conversacionales utilizados por los interlocutores (turnos, pares adyacentes, uso de reparaciones y/o de marcadores), 2) Estudiar cómo estos recursos puede influir en la estructura de la conversación y 3) Valorar el uso de otras formas de comunicación, como por ejemplo la gestualidad.

\section{Los informantes}

De acuerdo con los objetivos del presente estudio se seleccionaron informantes con diagnóstico de demencia tipo Alzheimer en fase inicial o media, pues durante estas etapas de la demencia la posibilidad de conversar y comprender el lenguaje se mantiene en un nivel que permite el poder participar en este tipo de intercambios. Los informantes debían cumplir con condiciones que permitieran filmar en sus hogares y además de esto, contar con el consentimiento informado tanto de la persona con Alzheimer (PA) como de su cuidador para participar en el estudio.

Se preseleccionaron diez familias, las cuales fueron visitadas para explicarles el alcance de la investigación. Sólo dos expresaron su deseo de ser parte de la investigación y dieron su consentimiento por escrito. Posteriormente se procedió a realizar dos filmaciones de prueba. Cabe destacar que se les informó a las cuidadoras que debían conversar abiertamente con su familiar con demencia de cualquier tema o tópico, ambas aceptaron las condiciones. Sin embargo y a pesar de que la inducción se mantuvo en ambos encuentros, se evidenció una notable dificultad en ellas para poder conversar de manera natural o espontánea con la PA.

Las cuidadoras primarias preferían hablar con la investigadora sobre los problemas de salud de sus familiares, incluso estando éstos presentes, y en numerosas ocasiones no permitían a la PA intervenir. Por esta razón la investigadora participó de manera activa y propició que la PA fuera incluida en la conversación. Esta situación se mantuvo a lo largo de los encuentros, por lo que se decidió analizar la participación de 
todos, a pesar de que este hecho se consideró como una limitación para el estudio y obligó a reconducir los objetivos del mismo.

De esta manera, la muestra de la presente investigación quedó constituida por dos personas con diagnóstico de enfermedad de Alzheimer (PA), sus cuidadoras primarias (CP) y la propia investigadora (I).

A continuación las características más importantes de las familias participantes.

\section{Familia $N^{o} 1$}

La PA es un hombre de 80 años con demencia tipo Alzheimer en fase intermedia. Vive con su esposa quien es su cuidadora primaria. Ambos están jubilados y su nivel económico se corresponde con el medio bajo. Comparten su vivienda con uno de sus hijos, la esposa de éste y una nieta de un año de edad. Este informante asiste periódicamente a sus controles en una Unidad de Memoria. Su esposa está dedicada exclusivamente a sus funciones como cuidadora. Ella es alegre, muy conversadora y a pesar del impacto de la enfermedad sobre sus vidas, se muestra optimista y emprendedora.

\section{Familia $N^{o} 2$}

La PA es una mujer de 67 años con demencia tipo Alzheimer en fase inicial, de nivel económico medio bajo, es bastante independiente, aún realiza labores del hogar y se comunica verbalmente de forma aceptable. Vive con su hija, pero sus días transcurren en la casa de su hermana menor quien es su cuidadora y vecina, ésta es una mujer de sesenta años, soltera, además es la cuidadora primaria de su madre, enferma y postrada en cama desde hace varios años. Esta doble función le genera cansancio y un mayor impacto. Su interacción con la informante es escasa, ella cree que su hermana no está bien, expresa que en "ocasiones dice incongruencias y pierde la cabeza" y que la responsabilidad de cuidarla le produce más trabajo.

\section{Elaboración del corpus y análisis de los datos}

Para elaborar el corpus del presente estudio, la investigadora visitó los hogares de las familias que aceptaron participar. Un técnico filmó las interacciones en cuatro ocasiones a lo largo de un año. Como ya se explicó previamente, a pesar de la inducción ofrecida a las cuidadoras, se dieron espacios de tiempo donde ambos no conversaron, las 
cuidadoras iniciaron poco la conversación con sus familiares, por lo que fue necesario que la I conversara activamente con la PA y con las CP.

En las conversaciones se abordaron diversos tópicos: tradiciones de la comunidad, historia de vida de la familia, fiestas religiosas y hechos contemporáneos de relevancia nacional. Es decir, en los cuatro encuentros con cada familia, se buscó construir un intercambio de información donde todos los interlocutores pudieran participar y ser activos en la construcción de la conversación. Para esto, la I inició el intercambio, generalmente con una pregunta o con un vocativo dirigido a la PA con la cual se introdujo un tema, y trató de mantener el interés en la conversación hasta lograr cumplir con el ciclo de apertura, toma de turnos y cierre.

El tipo de conversación elaborada fue del tipo cara a cara, los intercambios que la investigadora propuso se circunscribieron a un formato parecido al de la entrevista terapéutica, siguiendo la secuencia frecuente en los contextos logopédicos, en el cual se realizan diversas preguntas para iniciar la conversación.

El total de las filmaciones resultó en un video de 120 minutos aproximadamente. Posteriormente de este primer corpus se seleccionaron 20 eventos (10 por cada díada participante), se editaron y se trascribieron en glosa española. Estos eventos organizados en orden cronológico constituyeron los datos lingüísticos o corpus final de la investigación, posteriormente, estos datos se analizaron teniendo como guía los objetivos establecidos previamente para poder estudiar la relación entre los diferentes tipos de recursos utilizados por la $\mathrm{PA}$, la $\mathrm{CP}$ y por la propia investigadora, su impacto en la dinámica de la conversación, el uso de otros recursos comunicacionales y los cambios que el avance de la demencia producían en el discurso de la PA y en el de su CP.

Para la organización de los datos se partió de las notaciones propuestas por Levinson (1992, citado por Pietrosemoli 2007) de acuerdo con las características del corpus se utilizaron las siguientes marcas de transcripción:

I: investigadora

PA1: Persona con demencia Familia 1.

PA2: Persona con demencia Familia 2.

IC: Interlocutor clave, en este caso la cuidadora principal.

.... Silencio o pausa

: Repetición

_ Marcador discursivo. 
A continuación se presentan los resultados del estudio de algunas conversaciones. Por razones prácticas, se seleccionaron cuatro (dos por cada familia), atendiendo que el intercambio comunicativo se diera con una estructura completa, siguiendo la secuencia: inicio-desarrollo-conclusión, y además, en función de su extensión, de manera de contar con suficientes participaciones de todos los actores involucrados.

\section{Resultados}

Ejemplos de la Familia $\mathrm{N}^{\mathrm{o}} 1$

Evento 1

I: Señor N, ¿está comiendo bien?

PA1: ¿Comiendo?

I: Ujum (asiente con la cabeza)

PA1: Súper bien

I: Sí, ¿con buen apetito?

PA1: Eso sí.

I: Le están... ¿ está tomando alguna medicina?

PA1: Ella sabe, yo se, estoy bien, porque yo no tengo nada de malo, por aquí, por allá, por allá (mueve los brazos en varias direcciones y antes de responder mira a la cuidadora)

I: Y ¿está caminando?

PA1: Sí, me siento bien y todo.

I: ¿Usted sale a caminar con la señora L?

PA1: U:, hago lo que sea.

Comentarios: En este evento es posible identificar una repetición por parte de la PA1 (¿comiendo?) y una respuesta semejante a las de los pares adyacentes (súper bien), es decir, se contesta de forma casi obligada y coordinada a la pregunta (Pietrosemoli 2007). En la última parte del segmento, la selección por parte de la PA1 no se corresponde directamente con la estructura de par adyacente. En este caso, utilizó para iniciar su participación un conjunto de recursos: 1) mirar a su esposa y 2) afirmar que 
ella sabe. Posteriormente usó una sucesión de frases: por aquí, por allá, por allá, acompañada de gestos que parecen no relacionarse directamente con el tema. A continuación la I inició su intervención con el marcador $y$, seguido de la pregunta ¿está caminando? En la última participación la PA1 utilizó U: como una prolongación oralizada, que sugiere planificación o búsqueda de la respuesta correspondiente a la pregunta ¿usted sale a caminar con la señora $L$ ? o una pausa de hesitación.

\section{Evento 2}

I: Mire y cuénteme una cosa, ¿cómo se llama su hijo, el que es profesor de historia?

IC: El que vive aquí, ¿Cómo se llama el qué vive aquí?

PA1: A veces tengo una cosa aquí... (mueve las manos de forma circular como recordando)

I: ¿Y su nieta, cómo se llama su nieta, su última nieta?

IC: La bebecita de aquí

PA1: Eso es lo malo que tengo yo ahorita, que no me puedo acordar, eso, eso, sabes. (Señala y mira a su cuidadora)

Comentarios: Este evento se inició con un imperativo "mire", seguido del marcador "y", por parte de la investigadora quien comenzó el intercambio con una interrogante. La estructura de la pregunta presenta un elemento de especificación: "el que es profesor de historia", con el cual se trata de ayudar al paciente en la selección de la respuesta. Resalta la contribución que hace la cuidadora: "el que vive aqui", en búsqueda de lograr la comprensión de la PA1, utilizando la aclaratoria y repitiendo las preguntas de manera más específica. Estos recursos vuelven a ser utilizados por la investigadora y la cuidadora al plantearse una nueva interrogante. A pesar de sus esfuerzos, la PA1 no logró recordar los nombres solicitados. Sin embargo, fue capaz de explicar su dificultad para recordar, lo que evidencia que comprendió la exigencia del contexto, en este caso que debe responder una interrogante que se le está haciendo. Es necesario destacar el uso del marcador $y$ por parte de la investigadora, éste se presenta para la introducción de las preguntas, como recurso para captar la atención del informante. 
A continuación los ejemplos de la Familia $\mathrm{N}^{\mathrm{o}} 2$

\section{Evento 1:}

I: ¿Y qué hace aquí en su casa? Aquí en la casa de su mamá.

PA2: ¿Qué hago aquí?, nada que me pongo a ayudar a lavar los corotos, y si ayudar a barrer...no, ahorita no estoy barriendo así el patio por las piernas, porque me duele una pierna, me dijeron que no barriera...

I: ¿Y quién le dijo que no podía barrer?

PA2: ¿Ah?

I: ¿Quién le dijo que no barriera?

PA2: E:. el...el traumatólogo que me vio.

Comentario: En este evento puede apreciarse la repetición que hace la informante de la pregunta realizada por la investigadora. Sin embargo, inicia su respuesta con base a un listado de actividades en la cual aparece el marcador $y$ como elemento que ayuda a organizar la secuencia de sus actividades, y el uso de no como un recurso de rectificación para introducir a continuación una argumentación. Posteriormente, ante la confusión demostrada por la PA2 con el uso de ¿Ah?, es la investigadora quien debe repetir. Cabe destacar que en la última participación de la PA2, se observaron dos pausas continuas, antes de utilizar el término traumatólogo. Una vez más la investigadora utilizó el marcador $y$ al introducir una nueva pregunta, como recurso para captar la atención de la informante.

\section{Evento 2}

I: Mire, y ¿cómo va a hacer para ir a votar? Tiene que ir a votar, el 23 de noviembre hay elecciones.

PA2: ¡Sí!, voy con la andadera.

I: ¿Por quién va a votar?...si se puede saber.

IC: ¿Qué por quién vas a votar?

I: Ella me entendió perfecto (mirando directamente a la IC).

PA2: Nosotros siempre hemos...hemos sido adecos...

I: Sí...o sea, este, bueno ¿será quién?, será, ¿por Rosales?; ¿Rosales es adeco no? 
PA2: Por aquí los que no son de oposición son de ahí...son...son chavistas (Señala con los labios la casa de los vecinos)

I: ¿Usted cree que Santa Lucía es adeca? ¿Si?

PA2: ¡Éste año, vamos a ganar!, con el favor de Dios. (Hace seña positiva con su mano) Comentarios: En este evento la cuidadora interviene utilizando una repetición ¿Qué por quién vas a votar?, sin esperar que la PA2 responda, quizás tratando de asegurar que la PA2 haya entendido la pregunta. Por esta razón la investigadora interviene (sin solapar) y le asegura que la informante sí captó el mensaje. Cabe destacar que en su próxima intervención la PA2 no respondió directamente sino que inició su respuesta con un argumento con el cual expuso sus preferencias políticas: Nosotros siempre hemos...hemos sido adecos. En este evento se aprecia un tipo de preguntas diferentes, la investigadora percibe en la informante una mejor capacidad para llevar una conversación y por esto introduce un nuevo tema: ¿Usted cree que Santa Lucía es adeca?, interrogante que a pesar de que es cerrada, es respondida por la informante con una oración más compleja, es decir, aporta nueva información, amplía su respuesta y llega a expresar un deseo marcando su mensaje con un gesto afirmativo.

\section{Discusión}

Teniendo en cuenta los objetivos propuestos puede afirmarse que en la estructura de las conversaciones estudiadas los turnos se presentaron de forma ordenada y sincrónica, dándose escasas interrupciones. La duración de los turnos de la PA1 fueron más cortos, en muchas ocasiones solo utilizó una oración simple. Mientras que en la PA2 se dieron turnos más largos. La duración de la pausa se vio alterada en el caso de la PA1 por la participación de la IC, quien respondió por ella y en el caso de la PA2, cuando la IC repite una pregunta sin esperar que la PA2 responda. En la PA1 se observó también la concesión del turno a la IC buscando que la misma contestara las interrogantes.

Al emplear la investigadora el patrón típico de la entrevista terapéutica, se evidenció el uso frecuente de preguntas. Muchas de las interrogantes se correspondieron a las de tipo par adyacente, es decir las respuestas a las mismas pueden ser consideradas como previsibles, especialmente las preguntas con las cuales se inició la conversación. Ambas PA mostraron adecuación social en sus conversaciones pues sus respuestas se dieron de forma ajustada a lo esperado. Sin embargo, esto provocó una estructura conversacional donde no se favoreció que la PA pudiera iniciar los intercambios y en la cual los turnos se organizaran estrictamente. A este respecto cabe señalar que Gallardo 
Paúls (2007) advierte que la conversación con personas con Afasia muestra cambios en el ritmo conversacional y el uso estrategias de facilitación de la participación del paciente (preguntas polares, de inventario cerrado).

El uso de marcadores se presentó tanto en las PA como en la I, especialmente el marcador $y$ señalado por Pietrosemoli, Vera, González y Coutín (2005) como uno de los más utilizados en todos los contextos, incluso en los clínicos, específicamente con hablantes con Afasia. La I lo empleó para captar atención al iniciar sus turnos. En el caso de la PA el marcador y apareció como organizador y conector de secuencias. A este respecto es importante destacar que Pietrosemoli (2007) señala que aún en el caso de ser portadores de afecciones severas, los hablantes hacen un esfuerzo para conectar fragmentos de habla.

La ampliación de información fue utilizada por las cuidadoras y por la I, su uso podría relacionarse con las máximas de cantidad y de manera de Grice, en función de dar la información necesaria para exponer el tema. Sin embargo, también podría relacionarse con la intención de crear un mejor marco para el acceso al léxico por parte de la PA, es decir buscando ser más claro y evitar así la ambigüedad.

El uso de la gestualidad se presentó en la PA1, como una vía para responder a pesar de no poder acceder al léxico que demandó la situación. En la PA2 la gestualidad se vinculó más a la función de señalar un lugar o un objeto o a afirmar.

Cabe resaltar que la relación que estableció entre los actores estuvo marcada por una asimetría entre el actor-investigadora y los actores-paciente-cuidadora. Pues la necesidad de provocar la participación activa de las PA y de las CP definió el uso de interrogantes para generar las intervenciones. De esta forma, a la I le correspondió el turno predictivo e inicial de intercambio y a las $\mathrm{PA}$ y $\mathrm{CP}$, los turnos reactivos predichos (Gallardo-Paúls, 1998).

\section{Conclusiones}

Estos datos preliminares no pretenden ser concluyentes ni definir las características generales de la conversación en la persona que presenta demencia. El objetivo fundamental de este estudio es utilizar los fundamentos teóricos y metodológicos de la lingüística clínica para orientar la toma de decisiones y planificar la intervención fonoaudiológica teniendo datos más precisos sobre una función básica y fundamental, como lo es la conversación, teniendo en cuenta además, que el contexto de conversación en el hogar representa una situación muy distinta a la de los espacios clínicos, lugares 
donde tradicionalmente se ha estudiado la conversación de la persona con demencia en otras investigaciones (Hamilton, 1994; Sabat 2001; Brauner y Merel, 2006).

Sin embargo, es necesario destacar que la gestión de la conversación por parte del logopeda puede desplazar las características del contexto clínico a diferentes espacios, incluyendo el hogar de los pacientes, pues la manera como este profesional organiza la conversación puede influir en crear una interacción poco espontánea o natural. Por esto se propone investigar más ampliamente la actuación de todos los interlocutores en este contexto.

Los resultados de este estudio cualitativo, basado en una experiencia cotidiana y construida con actores familiares, pueden servir para orientar mejor a los logopedas para analizar la dinámica de la interacción con sus pacientes, tarea necesaria si se quiere reorientar el diseño de programas de estimulación lingüística en PA, y a cuidadores y familiares para desarrollar estrategias adecuadas para realmente favorezcan el intercambio con los pacientes. Es preciso insistir en la necesidad de seguir investigando en función de poder aclarar más y mejor, diversos aspectos de la actividad comunicativa de los diferentes actores y no perder de vista que los beneficios de este tipo de análisis se circunscriben a casos particulares. A pesar de esta limitación, una evaluación centrada en el estudio de la actividad lingüística de todos los actores permitirá una intervención lingüística más completa por parte de los especialistas.

\section{Referencias}

Aldana G. (2010). El cuidador de una persona con la enfermedad de Alzheimer: Cuando se debe cuidar a quien cuida. En Alzheimer: no estás solo. (pp.109-135). Caracas: Fundación Alzheimer de Venezuela-Capítulo Nueva Esparta.

Bayles, K., Tomoeda, C., Cruz, R., y Mahendra, N. (2000). Communication abilities of individuals with late-stage Alzheimer disease. Alzheimer Disease and Associated Disorders, 14, 176-181.

Brauner, D. y Merel, S. (2006). How a model base on linguistic theory can improve the assessment of decision-making for persons with dementia. The Journal of Clinical Ethics, 17, 139-148.

Brewer, J. (2005). Carrousel conversation: Aspects of family roles and topic shift in Alzheimer's talk. En Boyd H. Davis (ed.). Alzheimer talk, text and context, (pp87-101). New York: Palgrave MacMillan.

Díaz, F. (2010). Lenguaje espontáneo en el trabajo logopédico: un sencillo ideal y un 
producto interaccional. En B. Gallardo y V. Moreno (eds.), Estudios de lingüística clínica, V: Aplicaciones clínicas. Valencia: Universidad de Valencia.

Gallardo-Paúls, B. (1998). Comentario de textos conversacionales. De la teoría al comentario. Madrid: Arco Libros.

Gallardo-Paúls, B. (2005). Afasia y Conversación. Valencia: Tirant lo Blanch.

Gallardo-Paúls B. (2007). Pragmática para Logopedas. Cádiz: Publicaciones de la Universidad de Cádiz.

Gaona, C. (2012). El cerebro es nuestro mejor aliado de la comunicación y la felicidad. En La Comunicación en Parkinson y Demencia: ¿qué hacer? Universidad de Los Andes. Facultad de Humanidades y Educación. Centro de Investigación y Atención Lingüística (CIAL).

Grice, P. (1975). Lógica y Conversación. En L. Valdés (ed.). La búsqueda del significado, (pp. 511-530). Madrid: Tecnos.

Hamilton, H.E. (1994). Conversations with an Alzheimer's Patient: An interactional Sociolinguistic Study. Cambridge: Cambridge University Press.

Hernández, C. y Gallardo-Paúls, B. (2010). Tres condiciones de empirismo para los hechos del lenguaje, de interés en un enfoque cognitivo. Revista Española de Lingüística, 40, 73-96.

Hernández, C. (2012). Sobre la dimensión simbólica del déficit verbal. Racionalidad, emoción y experiencia en Pragmática Clínica. Foro Hispánico: Revista Hispánica de Flandes y Holanda, 44, 47-66.

Jaramillo, J. (2010). Demencias: los problemas de lenguaje como hallazgos tempranos. Acta Neurológica Colombiana, 26, 101-111.

Lesser, R. y Milroy, L. (1993). Linguistics and aphasia. Psycholinguistic and pragmatic aspects of intervention. Londres: Longman.

Ludí, M.C. (2005). Envejecer en un contexto de (des)protección social. Claves problemáticas para pensar la intervención social. Argentina: Espacio Editorial. MacKay, A.P. (2007). Linguagem nas Demências. En A. MacKay, V. Asensio-Ferreira, y T. Ferri-Ferreira (Eds.), Afasias e Demências. Avaliação e Tratamento Fonoaudiológico. São Paulo: Santos Livraria Editora.

McKhann, M., Knopman, D.S., Chertkow, H., Hyman, B.T., Jack, C., Kawas, C. et al 
(2011). The diagnosis of dementia due to Alzheimer's disease: Recommendations from the National Institute on Aging-Alzheimer's Association workgroups on diagnostic guidelines for Alzheimer's disease. Alzheimer's Dementia, 7, 263-269.

Moreno, V. (2010). L“avaluació pragmática em afàsia. Estudios de Lingüística Clínica. Valencia: Universitàt de València.

Maestre, G.E. (2007). Descubriendo los rostros del Alzheimer: una ventana a la identidad perdida. Maracaibo: Colección Textos Universitarios de la Universidad del Zulia.

Malagón, C., Rodríguez, J., Hernández, J. y Pardo, R. (2005). Análisis del desempeño del lenguaje en sujetos con demencia tipo Alzheimer. Revista Facultad de Medicina, 53, 3-9.

Orange, J.B., Lubinski, R. y Higginbotham, M. (1996). Conversational repair by individuals with dementia of the Alzheimer's type. Journal of Speech and Hearing Research, 39, 881-895.

Petersen, R.C. (2004). Mild cognitive impairment as a diagnostic entity. Journal of Internal Medicine, 256, 183-194.

Pietrosemoli, L., Vera, M., González, S. y Coutín, P. (2005). Marcadores discursivos en hablantes sanos y afásicos: el caso especial de y. Boletín de Lingüistica, 17, 2530.

Pietrosemoli, L. (2007). Análisis del discurso en poblaciones especiales: la conversación con afásicos. En A. Bolívar (comp.), Análisis del discurso. Por qué y para qué. Caracas: Los Libros de El Nacional.

Ripich, D.N. y Terrell, B.Y. (1988). Patterns of discourse cohesion and coherence in Alzheimer's disease. Journal of Speech and Hearing Disorders, 53, 8-15.

Rumbos, H. y Valles, B. (2010). Análisis discursivo de los textos narrativos en el adulto mayor con queja de memoria. En B. Gallardo-Paúls y V. Moreno (eds.), Estudios de Lingüística Clínica: Aplicaciones Clínicas. Valencia: Publicaciones de la Universitat de Valencia.

Savundranayagam, M. Y., Hummert, M. L. y Montgomery, R. J. (2005). Investigating the Effects of Communication Problems on Caregiver Burden. Journal of Gerontology, 60, 48-55.

Sabat, S. (2001). The experience of Alzheimer's disease. Life through a tangled veil. Massachusetts: Blackwell Publishers Inc. 
Tinti, A. y Marogna, M. (2000). Reabilitare l'afasia: una questione di parole? www.aphasiaforum.com/Gengll.htm

Ulin, P, Robinson, E. y Tolley, E. (2006). Investigación aplicada en salud pública. Métodos cualitativos. Washington: Organización Panamericana de la Salud.

Tomoeda, C. (2001). Comprehensive assessment for dementia: A necessary for differential diagnosis and management. Seminars in Speech and Language, 22, 275-289.

Tusón, A. (2002). Análisis de la conversación: entre la estructura y el sentido. Estudios de Sociolingüística, 3, 133-153.

Valles, B. (2008). Retos, perspectivas y consideraciones éticas en torno a la evaluación del lenguaje en la persona con demencia. Asociación Brasilera de Fonoaudiología. 13, 89-96.

Valles, B. (2010). La atención del lenguaje y la comunicación en las personas con demencia tipo Alzheimer. En Alzheimer: no estás solo. (pp.211-235). Caracas: Fundación Alzheimer de Venezuela-Capítulo Nueva Esparta.

Valles, B. (2011). Representaciones y creencias en torno a la evaluación lingüística del adulto mayor con trastorno cognitivo leve. Revista de Investigación en Logopedia, 1, 12-34.

Young, M. y Howells, C. (2010). Comunicación y demencia. Nuevas perspectivas. Barcelona: Editorial UOC. 